\title{
PRESENCIA DE ESTRUCTURAS ESTELARES EN LOS ORBITOCLYPEIDAE (FORAMINIFERA)
}

\author{
Carlos FERRÁNDEZ CAÑADELL
}

Departamento de Geología Dinámica. Geofísical y Paleontologia, Facultad de Geología, Universidad de Barcelona, Zona Universitaria de Pedralbes, 08071Barcelona. e-mail: cferran@natura. geo.ub.es

Ferrández Cañadell, C. 1998. Presencia de estructuras estelares en los Orbitoclypeidae (Foraminífera). [Presence of stellar structures in the Orbitoclypeidae (Foraminifera)]. Revista Española de Paleontología, 13 (1), 37-41. ISSN 0213-6937.

\begin{abstract}
A detailed structural study on the genera Orbitoclypeus and Asterocyclina (Orbitoclypeidae) has revealed new features of the microspheric nepiont. In both genera, the initial spiral chambers of microspheric forms have a stellar structure, that is, they have differentiated dorsal and ventral sides, the ventral one being subdivided into a main chamberlet and a stellar chamberlet. This stellar structure indicates a relationship of the Orbitoclypeidae with asterigerinaceans. Therefore, the Orbitoclypeidae are placed into the superfamily Asterigerinacea, as a sister family of the Amphisteginidae. In consequence, Asterocyclina and Orbitoclypeus would be more closely related to Amphistegina than to Discocyclina.
\end{abstract}

Keywords: Orbitoclypeidae, Orbitoclypeus, Asterocyclina, Asterigerinacea, Eocene, Foraminifera, microspheric nepiont.

\section{RESUMEN}

El estudio morfoestructural detallado de los géneros Orbitoclypeus y Asterocyclina (Orbitoclypeidae) ha permitido detectar la presencia en ambos géneros de camarillas estelares en las primeras cámaras espirales de las formas microesféricas. Dichas camarillas son asimétricas según el plano ecuatorial, con una mitad dorsal simple y una mitad ventral (donde se sitúan las aberturas) subdividida parcialmente en una camarilla principal y una camarilla estelar. La presencia de cámaras de estructura estelar indica una relación con los asterigerináceos, por lo que se sitúan los Orbitoclypeidae dentro de la superfamilia Asterigerinacea, como familia hermana de Amphisteginidae. En consecuencia, Asterocyclina y Orbitoclypeus estarían mas estrechamente relacionados con Amphistegina que con Discocyclina.

Palabras clave: Orbitoclypeidae, Orbitoclypeus, Asterocyclina, Asterigerinacea, Eoceno, Foraminifera, nepionte microesférico.

\section{INTRODUCCIÓN}

Desde los trabajos de Brönnimann (1946, 1951), los ortofragmínidos se clasifican en dos familias. Brönnimann consideró las diferencias en el nepionte microesférico como carácter supragenérico. Basándose en este carácter, Brönnimann propuso primero (1946) una clasificación en dos subfamilias: Discocyclininae (con Discocyclina y Actinocyclina) y Orbitoclypeinae (con Asterocyclina y Orbitoclypeus) que, en un trabajo posterior (Brönnimann, 1951), elevó a nivel de familia, aunque cambiando el nombre de la segunda a Asterocyclinidae. Como consecuencia, los distintos autores hacen referencia a esta familia bien como Asterocyclinidae (e.g. Less, 1987; Loeblich y Tappan, 1987; FerrándezCañadell y Serra-Kiel, 1992), bien como Orbitoclypeidae (e.g. Neumann, 1972; Caudri, 1972; Brolsma, 1973; Sirotti, 1987). De acuerdo con los artículos 34a y 36a del Código Internacional de Nomenclatura Zoológica, el nombre correcto es el de Orbitoclypeidae Brönnimann (nom. transl.).

Brönnimann (1946) estableció las principales diferencias en el crecimiento inicial de las formas microesféricas de los Discocyclinidae y los Orbitoclypeidae, destacando la diferencia en el crecimiento transicional (desde el crecimiento espiralado hasta el 

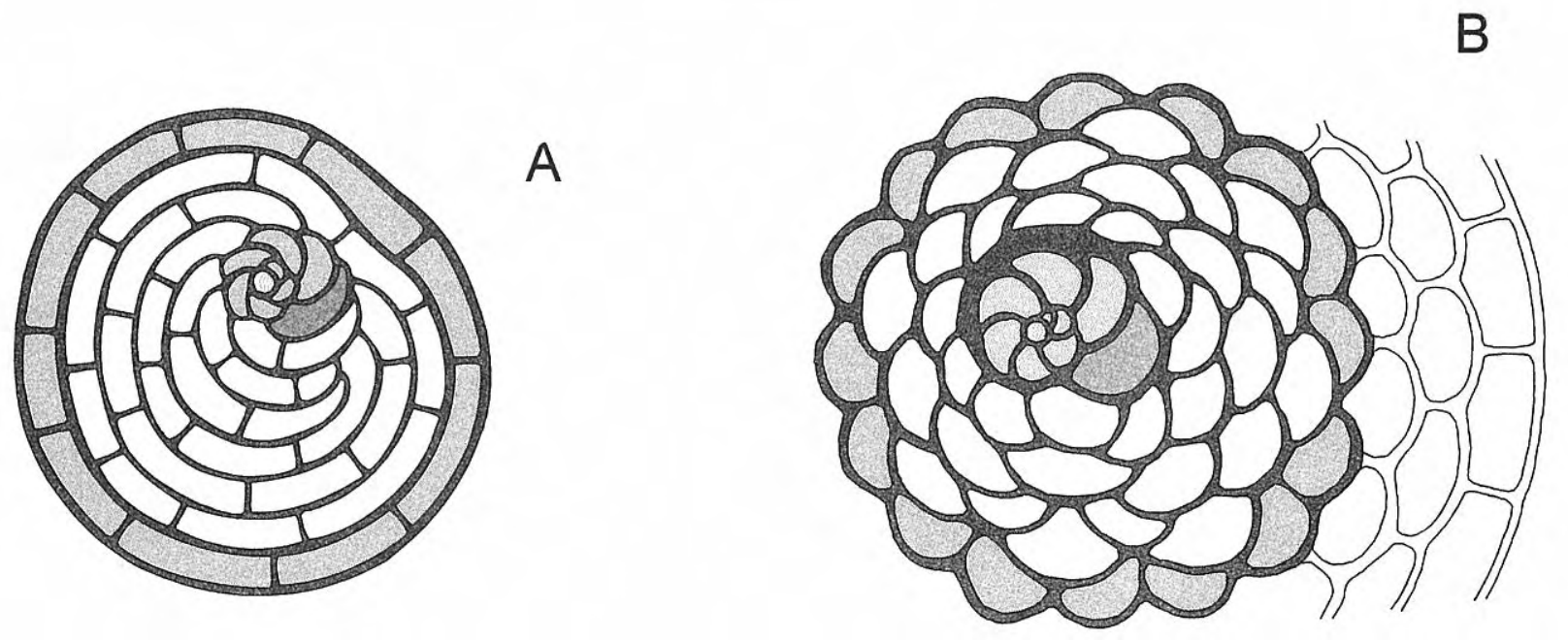

Figura 1. Esquema del nepionte microesférico en los ortofragmínidos, Discocyclinidae (A) y Orbitoclypeidae (B). En ambos grupos se pueden distinguir un estadio espiralado (en gris claro) y un estadio de transición (en blanco) hacia el crecimiento neánico, anular cíclico, del que se ha destacado en gris una cámara anular en cada esquema. La cámara de transición (en gris obscuro), la primera cámara con dos aberturas, marca el paso del estadio espiralado al de transición. En los Discocyclinidae (A), el estadio espiralado consta de cámaras sencillas, y el estadio transicional consiste en cámaras falciformes, subdivididas en camarillas mediante septos secundarios, que van creciendo en tamaño hasta formar un anillo completo e iniciar el estadio neánico. En los Orbitoclypeidae (B), el estadio espiralado se caracteriza por presentar una pared espiral engrosada y camarillas estelares; el estadio de transición consiste en cámaras de crecimiento orbitoidal, constituidas por camarillas aisladas que se van haciendo más numerosas hasta conformar un anillo completo (adaptado de Ferrández-Cañadell, 1995).

anular cíclico neánico): mientras en los Discocyclinidae consistiría en cámaras subdivididas en camarillas, en los Orbitoclypeidae las cámaras no estarían subdivididas. La ontogenia de las cámaras iniciales microesféricas de ambas familias fue explicada en más detalle por Ferrández-Cañadell (1995), que distinguió entre cámaras falciformes, subdivididas en camarillas mediante septos secundarios de lámina interna (séptulos), en los Discocyclinidae (ver también Ferrández-Cañadell et al., 1992), y camarillas orbitoidales aisladas, dispuestas en anillos, en los Orbitoclypeidae (Fig. 1). Esta distinción hace referencia sólo al crecimiento transicional del nepionte de las formas microesféricas. No obstante, algunos autores (e.g. Neumann, 1972; Loeblich y Tappan, 1987), probablemente malinterpretando las descripciones de Brönnimann (1946), extienden la diferencia al estadio neánico, aunque en ambas familias éste consiste en anillos subdivididos en camarillas rectangulares mediante septos secundarios de lámina interna (séptulos).

La ontogenia de las formas microesféricas de los discociclínidos, Discocyclina y Nemkovella, se explicó en trabajos anteriores (Ferrández-Cañadell et al., 1992; Ferrández-Cañadell y Serra-Kiel, 1992; FerrándezCañadell, 1997). En el presente trabajo se exponen los resultados del estudio morfoestructural detallado, mediante microscopio electrónico, del nepionte microesférico de los orbitoclipeidos, Orbitoclypeus y Asterocyclina, que han revelado nuevos caracteres estructurales.

\section{EL NEPIONTE MICROESFÉRICO DE LOS ORBITOCLYPEIDAE}

El crecimiento microesférico inicial se ha estudiado en las especies Orbitoclypeus douvillei (Schlumberger) y Asterocyclina stellata (d'Archiac) del yacimiento de Bosdarros (Cuenca de Aquitania, Francia), de edad Cuisiense inferior (Schaub, 1981). Ambas especies muestran el mismo tipo de estructura en las cámaras nepiónicas, difiriendo únicamente en el patrón estelar de los anillos en Asterocyclina.

El crecimiento de las formas microesféricas en los Orbitoclypeidae (Fig. 1B) se inicia con el prolóculo,

\section{Lámina I}

1-4 Orbitoclypeus douvillei (Schlumberger, 1903), Bosdarros, Cuisiense inferior. 1. Sección equatorial mostrando la mitad dorsal. 2. Sección equatorial mostrando la mitad ventral. 3, 4. Secciones equatoriales inclinadas, mostrando la estructura estelar de las cámaras espirales. p: camarilla principal; a: camarilla auxiliar; s: pared estelar.

5, 6 Asterocyclina stellata (d'Archiac, 1846), Bosdarros, Cuisiense inferior. Sección ecuatorial mostrando la mitad dorsal (5) y la mitad ventral (6) del mismo individuo. 
Lámina I

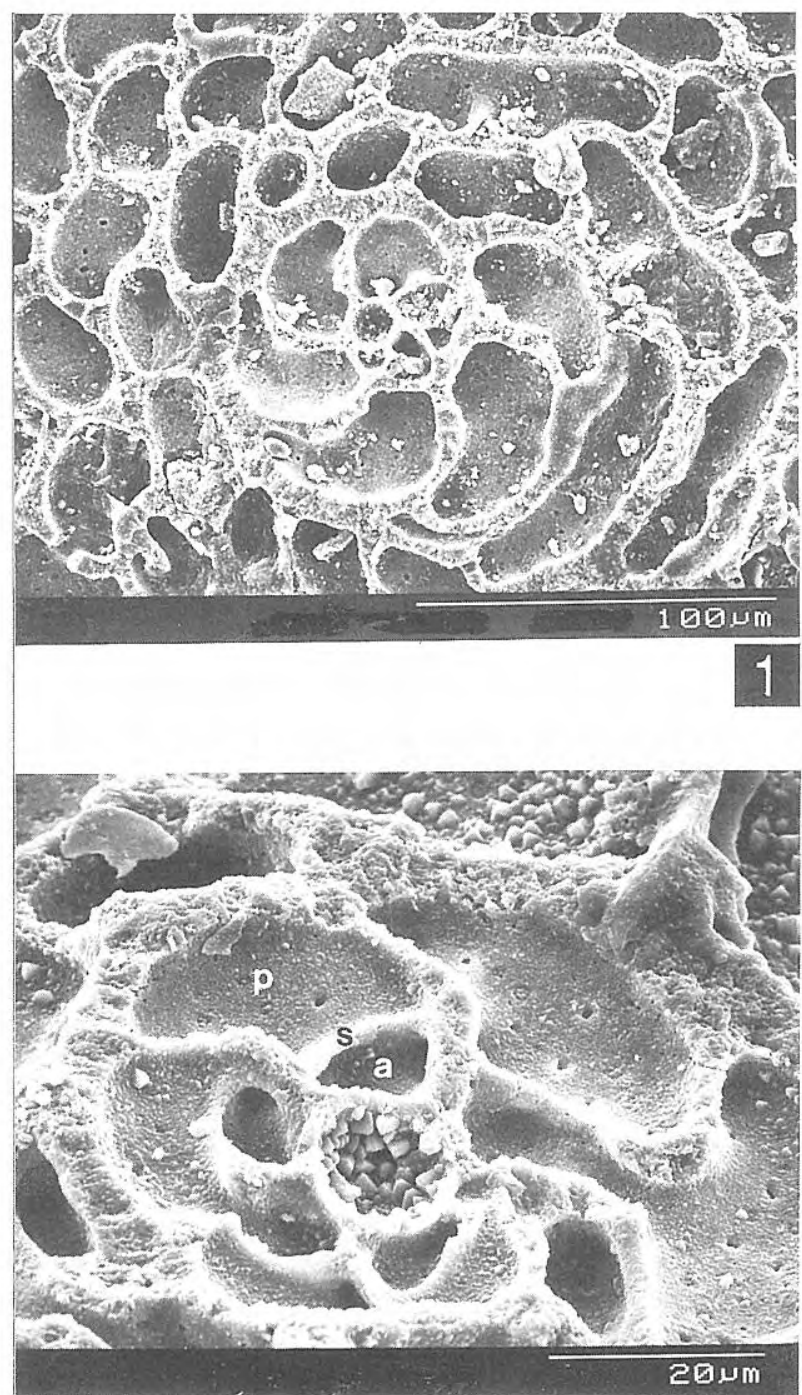

3

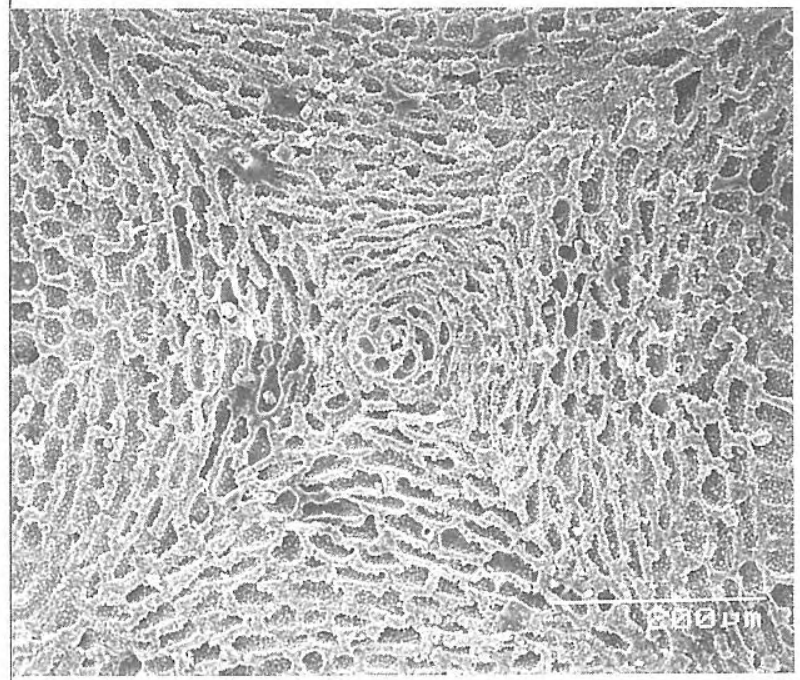

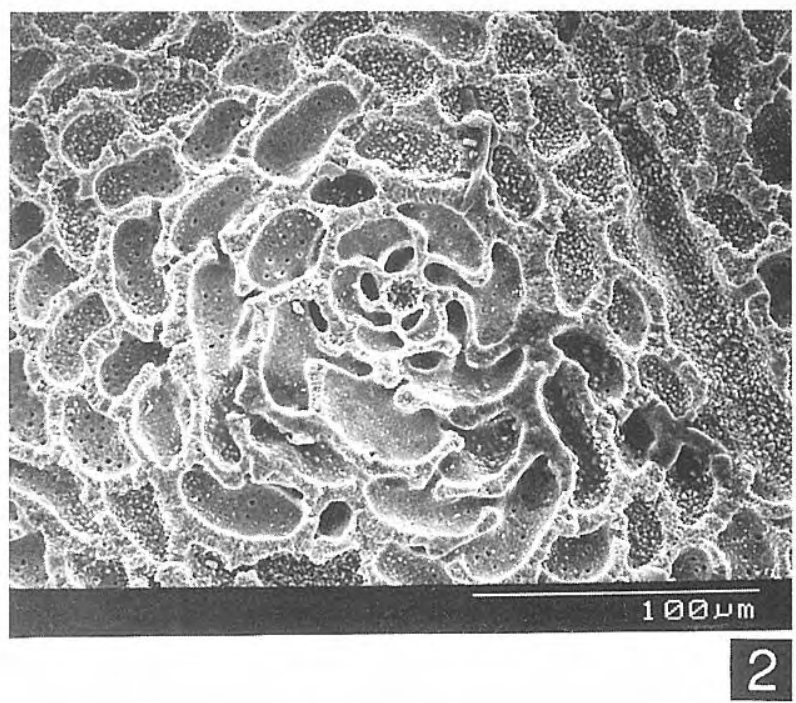
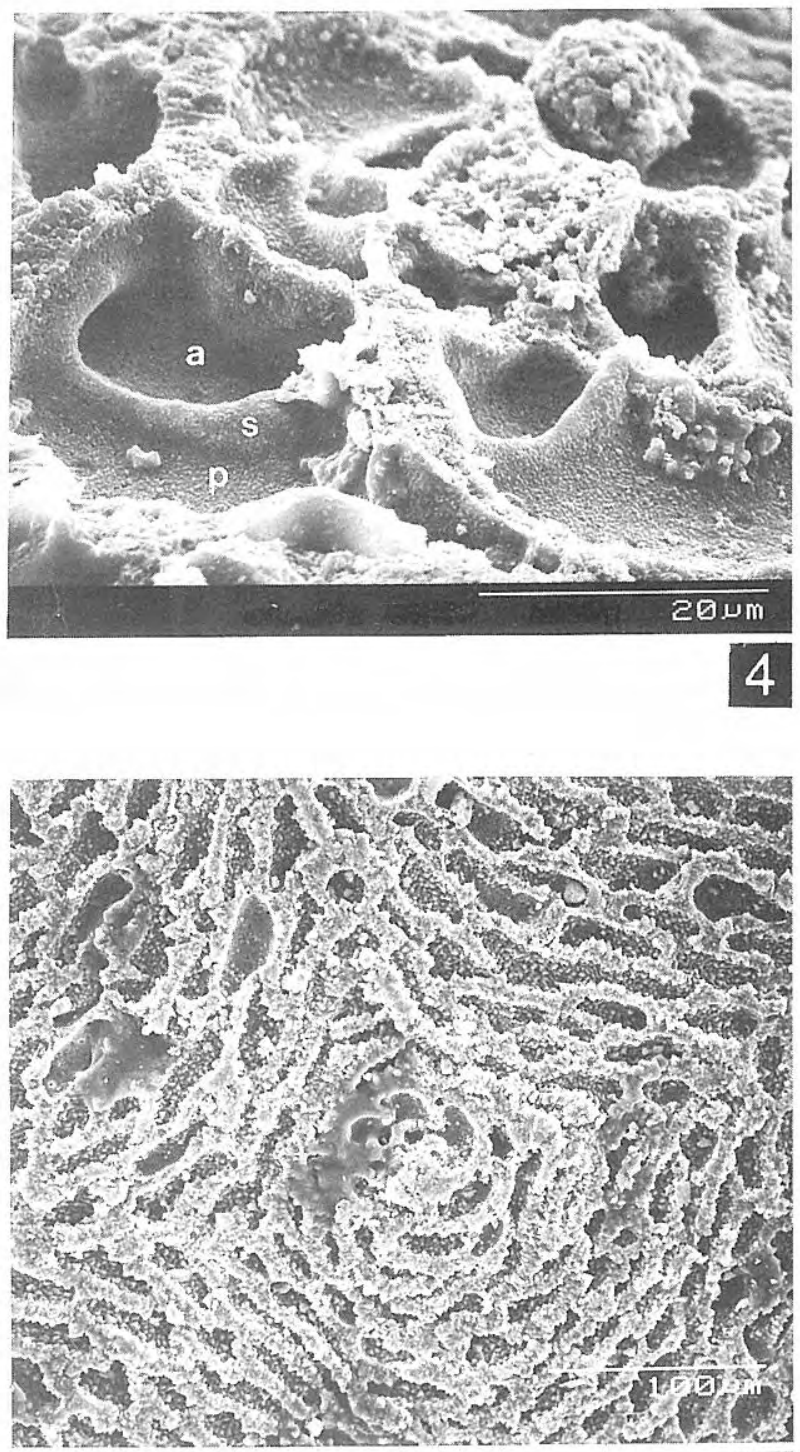
cámara inicial de morfología pseudoesférica cuya principal característica es la de no presentar poros. El diámetro del prolóculo es de 13-14 $\mu \mathrm{m}$ en las especies estudiadas, si bien varía entre 6 y $20 \mu \mathrm{m}$ en las especies de la Mesogea (Less, 1987). Al prolóculo sigue una serie de cámaras dispuestas planispiral-mente, conectadas una con otra mediante un solo estolón en posición proximal. La última cámara del estadio espiralado termina con una cámara con dos aberturas, la cámara de transición. La siguiente cámara, que inicia el estadio de transición, está constituida por dos camarillas arqueadas, formada cada una a partir de una de las aberturas de la cámara de transición. Ambas camarillas poseen dos aberturas proximales, una a cada lado, a partir de las que se forman nuevas camarillas en la siguiente cámara, siguiendo el patrón del crecimiento orbitoidal. La multiplicación de aberturas y camarillas lleva a la formación de un anillo. En las cámaras siguientes, las camarillas dispuestas en anillo van aumentando en longitud radial, aparecen septos secundarios de lámina interna y el septo pierde su contorno ondulado. Con el aplanamiento del septo, los estolones pasan a tener una dirección radial y aparecen las primeras aberturas oblicuas, a partir de las que se forman las primeras camarillas laterales.

En las especies estudiadas, la cámara de transición es la número 9-10. El número de cámaras espirales parece ser un carácter específico, distinto en las diferentes especies. Por otro lado, parece estar sujeto a una reducción filogenética, siguiendo la llamada aceleración nepiónica (Tan, 1935), ya que es menor en las especies estratigráficamente más altas de acuerdo con las descripciones de Less (1987). En Asterocyclina, el carácter estelar de los anillos se inicia muy temprano en la ontogenia de las formas microesféricas, en las primeras cámaras del estadio transicional orbitoidal.

Este tipo de crecimiento era ya más o menos conocido (Brönnimann, 1946; Sirotti, 1987). El estado excepcional de conservación del material estudiado, no recristalizado y vacío de cemento o sedimento, ha permitido descubrir nuevos caracteres en el nepionte de los orbitoclipeidos. En los dos géneros estudiados, Orbitoclypeus y Asterocyclina, las cámaras del estadio espiralado son asimétricas según el plano ecuatorial. Mientras la mitad dorsal de las cámaras espirales es sencilla, la mitad ventral (donde se sitúan las aberturas, posteriormente transformadas en estolones) está subdividida en dos partes mediante un septo secundario parcial de lámina interna (Lám. I, figs. 1-6). Dada la similitud de esta morfoestructura con la de los asterigerináceos (como Asterigerina), emplearemos la terminología utilizada en la descripción de este grupo. Así, el estadio espiralado de las formas microesféricas de los Orbitoclypeidae está constituido por cámaras estelares, con una mitad dorsal y una ventral disferenciadas. La mitad ventral (Lám. I, figs. 2, 3, 4, 6) está subdividida en una camarilla principal y una camarilla estelar mediante una pared estelar de lámina interna. La camarilla estelar puede ser simplemente una continuación de la camarilla principal o puede estar más desarrollada en sentido ventral (Lám. I, figs. 3, 4). Los estolones se sitúan en la parte proximal (umbilical) de la pared de las camarillas estelares, de manera que la camarilla estelar de una cámara comunica con la camarilla principal de la cámara siguiente (Lám. I, figs. 2, 3, 4). La estructura estelar se encuentra normalmente sólo en las cámaras del estadio espiralado, aunque se han podido observar algunas pequeñas camarillas estelares en las primeras camarillas del estadio transicional en $O$. douvillei.

Una revisión de la bibliografía ha permitido detectar estructuras estelares en otras especies de orbitoclypeididos. Por un lado, pueden observarse en las fotografías de Orbitoclypeus nummuliticus (Gümbel) del Luteciense superior de Verona (Italia), publicadas por Sirotti (1987). En la Lám. 2, fig. 3 del trabajo de Sirotti, pueden observarse claramente paredes estelares en las cámaras tercera a sexta (en ese espécimen la cámara de transición es la octava, no la quinta como interpreta Sirotti en su Figura 4). Por otro lado, en la detallada descripción de la forma microesférica de "Discocyclina" anconensis Barker, del Eoceno Medio de Barbados, Vaughan (1945: p. 33) describe subdivisiones en las cámaras espirales, que pueden interpretarse como estructuras estelares. Esta especie fue posteriormente tomada por Caudri (1972) como especie tipo para el nuevo género Neodiscocyclina, homólogo americano de Orbitoclypeus, del que se diferencia por presentar estolones anulares. Las estructuras estelares, por tanto, se encuentran en los Orbitoclypeidae de la Mesogea y de la bioprovincia americana.

\section{DISCUSIÓN}

El tipo de crecimiento del nepionte microesférico de los macroforaminíferos, comunmente distinto del crecimiento neánico, se interpreta como un vestigio de la forma ancestral de la que se originaron (e.g. Douvillé, 1906; van der Vlerk y Gloor, 1968; Brasier, 1984). Siguiendo esta interpretación, pueden considerarse los Orbitoclypeidae como originados a partir de un antecesor asterigerináceo, de los que se conocen formas cretácicas y paleocenas (Loeblich y Tappan, 1987). Dada esta relación de los Orbitoclypeidae con los asterigerináceos, se propone incluirlos en la superfamilia Asterigerinacea, del mismo modo que se incluye la familia monogenérica Amphisteginidae. Una consecuencia implícita de esta interpretación es que los orbitoclipeidos (Asterocyclina, Orbitoclypeus y Neodiscocyclina) estarían más estrechamente relacionados con el género Amphistegina, que también presenta cámaras estelares en el nepionte microesférico, muy semejantes a las de los orbitoclipeidos (ver la Lám. 88, fig. 4 de Hottinger et al., 1993), que con los Discocyclinidae.

\section{CONCLUSIONES}

Los ortofragmínidos orbitoclipeidos (Asterocyclina, Orbitoclypeus y Neodiscocyclina) presentan cámaras 
estelares en el nepionte microesférico. Se consideran, por tanto, relacionados filogenéticamente con los asterigerináceos, y se sitúa la familia Orbitoclypeidae dentro de la superfamilia Asterigerinacea. Como consecuencia, los orbitoclipeidos, como Asterocyclina, estarían mas estrechamente relacionados con Amphistegina que con los discociclínidos.

\section{AGRADECIMIENTOS}

El autor expresa su agradecimiento al Prof. L. Hottinger, de la Universidad de Basilea, por haber facilitado parte del material estudiado y por la discusión crítica del trabajo, y al personal del Servicio de Microscopía Electrónica de la Universidad Central de Barcelona. Este trabajo ha recibido soporte económico de una beca postdoctoral F.P.U. del M.E.C. y del proyecto de investigación de la DGICYT PB95-0883.

\section{BIBLIOGRAFÍA}

Brasier, M. 1984. Discospirina and the pattern of evolution in foraminiferid architecture. Benthos'83, 2nd International Symposium on Benthic Foraminifera, (Pau, April 1983), Elf Aquitaine, Esso REP, Total CFP, Pau et Bordeaux, 87-90.

Brolsma, M.J. 1973. Biometric data of Discocyclinids from Early Eocene deposits of the Tuilerie de Gan, France. Proceedings of the Koninklijke Nederlandse Akademie van Wetenschappen, ser. B, 76, 411-422.

Brönnimann, P. 1946. Zur Frage der verwandtschaftlichen Beziehungen zwischen Discocyclina s.s. und Asterocyclina. Eclogae geologicae Helvetiae, 38(2), 579615.

Brönnimann, P. 1951. A model of the internal structure of Discocyclina s. str. Journal of Paleontology, 25, 208211.

Caudri, C. B. M. 1972. Systematics of the American Discocyclinas. Eclogae geologicae Helvetiae, 65(1), 211-219.

Douvillé, H. 1906. Évolution et enchaînement des Foraminifères. Bulletin de la Société Géologique de France, sér. 4, 6(7), 588-602.

Ferrández-Cañadell, C. 1995. Morfostructura i Paleobiologia dels Ortofragmínids de la Mesogea (Discocyclinidae $i$ Asterocyclinidae, Foraminifera). Tesis Doctoral, inédita, Universitat de Barcelona, $271 \mathrm{pp}$.

Ferrández-Cañadell, C. 1997. A new, ribbed, species of Nemkovella Less, 1987 (Discocyclinidae), and discussion on the genus Actinocyclina Gümbel, 1870. Journal of Foraminiferal Research, 27(3), 175-185.

Ferrández-Cañadell, C. and Serra-Kiel, J. 1992. Morphostructure and paleobiology of Discocyclina Gümbel, 1870. Journal of Foraminiferal Research, 22(2), 147-165.

Ferrández Cañadell, C., López Civit, C. y Serra-Kiel, J. 1992. Estructura y ontogenia del género Discocyclina Gümbel, 1870 (macroforaminífero del Eoceno). Revista Española de Paleontología, 7, 13-23.

Hottinger, L., Halicz, E. and Reiss, Z. 1993. Recent Foraminiferida from the Gulf of Aqaba, Red Sea. Academia Scientiarum et Artium Slovenica, Classis IV. Historia Naturalis, 33, 1-179.

Less, G. 1987. Paleontology and stratigraphy of the european orthophragminae. Geologica Hungarica, Series Paleontologica, 51, 49-373.

Loeblich, A.R., Jr. and Tappan, H. 1987. Foraminiferal Genera and Their Classification. Van Nostrand Reinhold Company, New York, 2 volumes, 970 pp. + 212 pp., 847 pl.

Neumann, M. 1972. Sur les orbitoïdés du Crétacé supérieur et du Tertiaire, II, structure et classification. Revue de Micropaléontologie, 15(3), 163-189.

Schaub, H. 1981, Nummulites et Assilina de la Téthys paléogène. Taxinomie, phylogenèse et biostratigraphie. Memoires Suisses de Paléontologie, 104, 105, 106, 236 pp.

Sirotti, A. 1987. Ontogeny in Tethyan Discocyclinidae and Orbitoclypeidae (Foraminifera). Bolletino della Società Paleontologica Italiana, 26(3), 269-278.

Tan, Sin Hok. 1935. Die peri-embryonalen Äquatorialkammern bei einigen Orbitoiden. De Ingenieur in Nederlandsch-Indië, 2(12), 113-126.

Vaughan, T.W. 1945. American Paleocene and Eocene larger foraminifera. In: American Old and Middle Tertiary Larger Foraminifera and Corals. Memoirs of the Geological Society of America, 9(1), 1-175.

Vlerk, I.M. van der and Gloor, H. 1968. Evolution of an embryo. Genetica, 39, 45-63. 